\title{
The effect of dicyclohexylamine and fumagillin on Nosema ceranae-infected honey bee (Apis mellifera) mortality in cage trial assays
}

\author{
Johan P. van den HeEver ${ }^{1}$, Thomas S. Thompson ${ }^{1}$, Simon J. G. OtTo ${ }^{1,2}$, \\ Jonathan M. Curtis ${ }^{3}$, Abdullah IBrahim ${ }^{4}$, Stephen F. PernaL ${ }^{4}$ \\ ${ }^{1}$ Alberta Agriculture and Forestry, Food Safety Division, Agri-Food Laboratories Branch, 6909-116 Street, Edmonton, \\ Alberta T6H 4P2, Canada \\ ${ }^{2}$ School of Public Health, University of Alberta, Edmonton, Alberta T6G 1C9, Canada \\ ${ }^{3}$ Department of Agricultural, Food and Nutritional Science, University of Alberta, Edmonton, Alberta T6G 2P5, Canada \\ ${ }^{4}$ Agriculture and Agri-Food Canada, Beaverlodge Research Farm, P.O. Box 29, Beaverlodge, Alberta T0H 0C0, Canada
}

Received 24 June 2015 - Revised 1 October 2015 - Accepted 5 November 2015

\begin{abstract}
Both commercially available fumagillin-based treatments for honey bees (Apis mellifera), Fumagilin$\mathrm{B}^{\mathbb{B}}$ as well as Fumidil- $\mathrm{B}^{\mathbb{B}}$, contain the reportedly genotoxic and tumorigenic compound dicyclohexylamine (DCH) as the counter ion in the fumagillin-DCH salt. The effect of DCH, purified fumagillin (containing no DCH), and the commercial formulation Fumagilin- $\mathrm{B}^{\mathbb{B}}$ (containing both fumagillin as well as $\mathrm{DCH}$ ) on the mortality of caged Nosema ceranae-infected honey bees was investigated. A statistically significant risk of bee mortality associated with oral exposure to $\mathrm{DCH}$ was observed. $\mathrm{DCH}$ is also known to be significantly more stable than fumagillin in honey under a variety of temperature conditions, both in the presence and absence of light. The presence of DCH in the hive is therefore a potential concern for bee health and also for food safety.
\end{abstract}

\section{Fumagilin-B ${ }^{\circledR}$ / fumagillin / dicyclohexylamine / DCH / toxicity / honey}

\section{INTRODUCTION}

Fumagillin (Figure 1) was discovered nearly 70 years ago (Hanson and Eble 1949, Eble and Hanson 1951). and its efficacy against Nosema apis infections (Zander 1909) plaguing the honey bee (Apis mellifera L.) was soon realized (Katznelson and Jamieson 1952, Bailey 1953). The more recently reported Nosema ceranae (Fries et al. 1996) infection of the western honey bee forms part of the pathogen complex collectively referred to as "nosema disease." N. apis and $N$. ceranae are distinctly different single-cellular

Corresponding author: J. van den Heever, johan.vandenheever@gov.ab.ca Manuscript editor: Monique Gauthier microsporidian fungal parasites which have been associated with high levels of bee loss worldwide, with both $N$. apis and $N$. ceranae being implicated as part of the pathogen complex associated with the colony collapse disorder (CCD) phenomenon (Cox-Foster et al. 2007; Martín-Hernández et al. 2007; Higes et al. 2008, 2009; vanEngelsdorp et al. 2009). Currently, Fumagilin- $\mathrm{B}^{\circledR}$ is the only registered antibiotic available to treat both $N$. apis and $N$. ceranae infections of honey bees in North America, although it is reportedly not as effective against $N$. ceranae (Williams et al. 2008, 2011). Fumagillin, sold as Fumidil- $\mathrm{B}^{\circledR}$ in Europe, is reportedly only allowed for use under special circumstances in parts of Europe, including Spain and some Balkan countries (Higes et al. 2011, Stevanovic et al. 2013). and is not available for general use. 


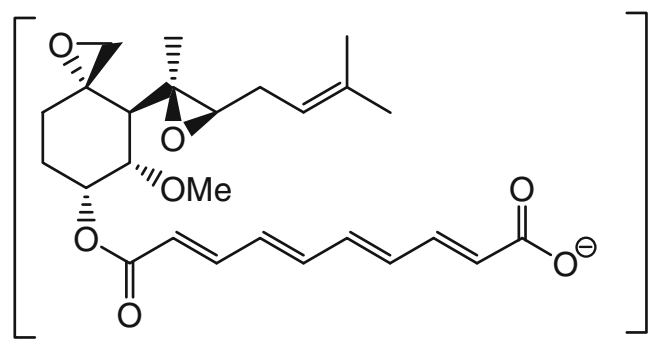

a

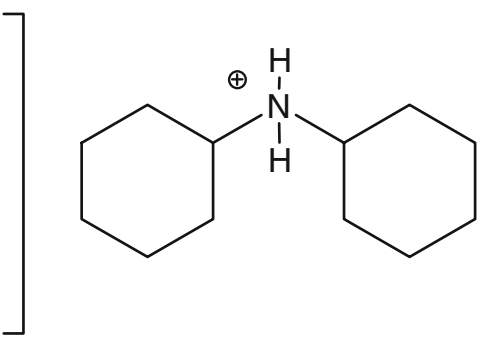

b

Figure 1. The commercial Fumagilin- $\mathrm{B}^{\circledR}$ (or Fumidil- $\mathrm{B}^{\circledR}$ ) containing fumagillin (a) as the dicyclohexylamine (b) salt.

A review paper (van den Heever et al. 2014) recently pointed out that the commercial formulations of fumagillin contain fumagillin as a salt, with dicyclohexylamine ( $\mathrm{DCH}$ ) being the counter ion of fumagillin in this salt (Figure 1). It is therefore important to realize that both fumagillin and $\mathrm{DCH}$ are present in a 1:1 stoichiometric ratio when applying either of the commercial formulations. DCH is reportedly genotoxic (Stanimirović et al. 2010) as well as tumorigenic (Sigma-Aldrich MSDS 185841 v. 5.0 Rev. 07/24/2012), and application of either Fumagilin-B ${ }^{\circledR}$ or Fumidil-B ${ }^{\circledR}$ implies that the same amount of the biologically active DCH is also being applied at the same time. For a more comprehensive discussion on the toxicity of fumagillin and $\mathrm{DCH}$, the reader is referred to a recent review (van den Heever et al. 2014). During our previous research using $N$. ceranaeinfected caged bees to evaluate alternative chemotherapies for use against this parasite, we observed bee mortalities in the positive control cages being treated with Fumagilin-B ${ }^{\circledR}$ of up to $71 \%$ (van den heever et al. 2015c). In these trials, the treatment dose was $40 \mu \mathrm{M}$ in $60 \%$ sucrose syrup solution. The observed mortality rates also increased with an increase in Fumagilin- $B^{\circledR}$ concentration. It was therefore suspected that the elevated bee mortality associated with Fumagilin-B ${ }^{\circledR}$ usage could be ascribed to the presence of either DCH or fumagillin in the commercial products. The present study was thus undertaken to evaluate changes in adult bee survival and $N$. ceranae treatment efficacy associated with feeding caged bees with $\mathrm{DCH}$ only (no fumagillin), pure fumagillin (no DCH), as well as with the commercially available
Fumagilin-B ${ }^{\circledR}$, consisting of both fumagillin as well as DCH.

\section{MATERIALS AND METHODS}

\subsection{Reagents and materials}

Pure fumagillin isolated from Aspergillus fumigatus containing no DCH (Cat. \# F6771), and dicyclohexylamine nitrite (Cat. \# 317837) was obtained from Sigma-Aldrich (St. Louis, MO, USA). The commercial formulation of fumagillin, Fumagilin- $B^{\circledR}$, containing fumagillin as the DCH salt, was obtained from Medivet Pharmaceuticals Ltd (High River, AB, Canada; DIN 02231180).

\subsection{Cage assays}

Cage assays were conducted during 2013. Adult honey bees (A. mellifera) for the assays were obtained from several colonies at Agriculture and Agri-Food Canada's Research

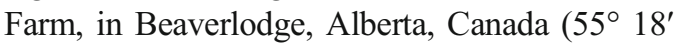
$\mathrm{N} ; 119^{\circ} 17^{\prime} \mathrm{W}$ ) by collecting frames of sealed brood with newly eclosing bees. These colonies were repeatedly tested in order to establish whether they were free from both $N$. apis and $N$. ceranae infections, using both light microscopic and molecular methods (described below). Frames were kept overnight in an incubator (Percival Model 136NLC9, Percival Scientific Inc., Perry, IA, USA) maintained at hive temperature $\left(33 \pm 0.5^{\circ} \mathrm{C}\right)$ and relative humidity $(70 \pm 5 \%)$. Adult workers were pooled and mixed from 
all frames, with 100 being added to wooden screened cages $(8.0 \times 9.5 \times 12.0 \mathrm{~cm}$ ID) for the cage assay. Bees were then fed $4 \mathrm{~mL}$ of a $60 \%$ $(w / v)$ of aqueous sucrose syrup for $24 \mathrm{~h}$, using gravity feeders made from disposable centrifuge tubes (Cat. \#93000-020, VWR International, Radnor, PA, USA).

After the initial 24-h feeding period with the $60 \%$ sugar syrup, each cage was mass inoculated with $5 \mathrm{~mL}$ of a $60 \%$ syrup solution containing $1 \times$ $10^{7}$ freshly harvested $N$. ceranae spores. Spores were prepared from previously identified colonies of honey bees with established high levels of $N$. ceranae infection. Workers from these colonies were euthanized on dry ice, followed by removal of their abdomens, which were then suspended in ultrapure water (1 mL per bee). After maceration, the crude suspension was filtered through a sieve $(\sim 0.8 \mathrm{~mm})$ in order to remove large body parts, and the resulting crude macerate was then counted, according to methods below, in order to prepare a solution with the correct inoculation dose. Some of the crude macerate was frozen for use in subsequent Nosema spp. identification. After consumption of the inoculum for $48 \mathrm{~h}$, cages of bees were fed ad libitum for 17 days with the three test compounds, or groups of compounds, consisting of fumagillin only, DCH only, as well as the commercially available Fumagilin- $\mathrm{B}^{\circledR}$ (fumagillin and $\mathrm{DCH}$ ), all at a single concentration of $40 \mu \mathrm{M}$ in $60 \%$ sucrose syrup. We were only interested in evaluating the label-dose effects, and therefore, only a single test concentration was used. A negative control consisting of a $60 \%$ sucrose solution was also employed. Six replicate cages of bees were evaluated for each control, compound, or mixture of compounds, and the mortality of bees was recorded each day, up to 17 days postinoculation.

\subsection{Determination of spore levels}

To determine Nosema infection levels in colonies, 60 adult workers were collected from peripheral frames of the brood nest. For cage trials, 30 surviving workers were removed from each cage 17 days post-inoculation. Bees were euthanized and had their abdomens placed into a stomacher bag containing $70 \%$ ethanol ( $1 \mathrm{~mL}$ per bee). The abdomens were then macerated for $1 \mathrm{~min}$ at medium speed (Seward Stomacher ${ }^{\circledR} 80$ Biomaster, Seward Laboratory Systems Inc., Davie, FL, USA), and $6 \mu \mathrm{L}$ of the macerate was withdrawn and loaded onto a Helber Z30000 counting chamber (Hawksley, Lancing, UK), with spores counted according to the generalized methods of Cantwell (1970) under phase contrast microscopy at $\times 400$ magnification. Samples of the remaining crude macerate were portioned into $1.5-\mathrm{mL}$ microcentrifuge tubes and stored at $-20^{\circ} \mathrm{C}$.

\subsection{Nosema spp. identification}

The crude macerate described in Section 2.3 was vortexed, and then $200-400 \mu \mathrm{L}$ was centrifuged to remove the ethanol from the sample. DNA extraction was performed using the DNeasy ${ }^{\circledR}$ Blood \& Tissue Kit (Qiagen ${ }^{\circledR}$, Valencia, CA, USA). The concentration of the extracted DNA was determined spectrophotometrically (NanoDrop 2000C, Thermo Scientific, West Palm Beach, FL, USA), whereafter 50$100 \mathrm{ng}$ of this DNA extract was amplified using polymerase chain reactions (PCR).

A multiplex system that co-amplified the $16 \mathrm{~S}$ rRNA gene of $N$. apis and $N$. ceranae (MartínHernández et al. 2007) as well as the honey bee ribosomal protein RpS5 gene (Thompson et al. 2007) was used within the same reaction. A modified version of the PCR protocol was used, owing to the fact that early pre-tests indicated that these modifications increased the sensitivity of simultaneous detection of both $N$. apis and $N$. ceranae within any given sample. All PCR reactions were performed using a Mastercycler ${ }^{\circledR}$ proS thermocycler (Eppendorf, Mississauga, Canada) and utilizing the Illustra ${ }^{\mathrm{TM}}$ PuReTaq Ready-ToGo $^{\text {TM }}$ PCR beads (GE Healthcare Life Sciences, Baie d'Urfe, Quebec, Canada). PCR beads were reconstituted to $25 \mu \mathrm{L}$ final volume by adding sterile $\mathrm{H}_{2} \mathrm{O}, 0.5 \mu \mathrm{L}$ of $20 \mathrm{mM}$ forward and reverse primers (a final concentration of $0.4 \mathrm{mM}$ ), and the DNA (50-100 ng per reaction). To amplify a 218-bp 16S rRNA PCR product specific for $N$. ceranae, primers Mitoc-For (5'-CGGCGAC GATGTGATATGAAAATATTAA-3') and MitocRev (5'-CCCGGTCATTCTCAAACAAAAAA 
CCG-3') were used, and to amplify a 321-bp 16S rRNA PCR product specific for $N$. apis, primers A p is-For ( $5^{\prime}-$ G G G G G C A T G T C T T T GACGTACTATGTA-3') and Apis-Rev (5'GGGGGGCGTTTAAAATGTGAAACAACTATG-3) were used, according to Martín-Hernández et al. (2007). In addition, the honey bee housekeeping gene, RpS5, was also amplified within the same reaction as a reference, which yielded a PCR product of $115 \mathrm{bp}$. The primer pairs used were RpS5-For (5'-AATTATTTGGTCGCTGG AATTG-3') and RpS5-Rev (5'-TAACGTCCAG CAGAATGTGGTA-3'), respectively (Thompson et al. 2007). The thermocycler program consisted of an initial DNA denaturation step at $95{ }^{\circ} \mathrm{C}$ for $5 \mathrm{~min}$, followed by 35 cycles of $94{ }^{\circ} \mathrm{C}$ for $30 \mathrm{~s}$, $61.8{ }^{\circ} \mathrm{C}$ for $30 \mathrm{~s}$, and $72{ }^{\circ} \mathrm{C}$ for $30 \mathrm{~s}$. A final extension of $72{ }^{\circ} \mathrm{C}$ for $7 \mathrm{~min}$ was followed by holding the reactions at $4{ }^{\circ} \mathrm{C}$ until stopped. All PCR products were visualized on a $2 \%$ agarose gel and stained with SYBR ${ }^{\circledR}$ Safe DNA gel stain (Life Technologies, Carlsbad, CA, USA).

\subsection{Statistical analysis}

Data was analyzed using semi-parametric, Cox proportional hazards models with a complimentary log-log link for survival data (Corrente et al. 2003, Dohoo et al. 2009). The bee survival for indicator variables for each treatment group was considered against negative controls using likelihood ratio test (LRT; $P \leq 0.05$ ). To test for proportional hazards across treatment groups, an interaction between treatment group and survival time was considered. A LRT $P \leq 0.05$ indicated non-proportional hazards, which was solved by including the interaction in the model. Linear regression was used to assess the effects of these treatment methods on the natural log of spore counts to normalize the data (zero spore counts were changed to one to allow for the log value to be zero). The effect of an indicator variable treatment group was tested using the extra sum of squares F-test (Dohoo et al. 2009). The model was assessed for fitness by evaluating the normality and homoscedasticity of the standardized residuals. All analysis was conducted in Excel 2010 (Microsoft Corporation, Redmond, WA, USA) and STATA Intercooled 13.1 (StataCorp LP, College Station, TX, USA).

\section{RESULTS}

The Cox proportional hazards model found that the treatment group and an interaction between the treatment group and survival time were significant (LRT $P<0.01$ for both). This indicated that the hazards for each treatment group varied over time.

The predicted hazards by treatment group are shown in Figure 2. Specific comparisons between two treatments at distinct time points (hazard ratios) are shown in Table I. For the first 10 days of the trial, there were no obvious differences in the hazards (probability of death) for any treatment group. Starting on day 11 , the commercial

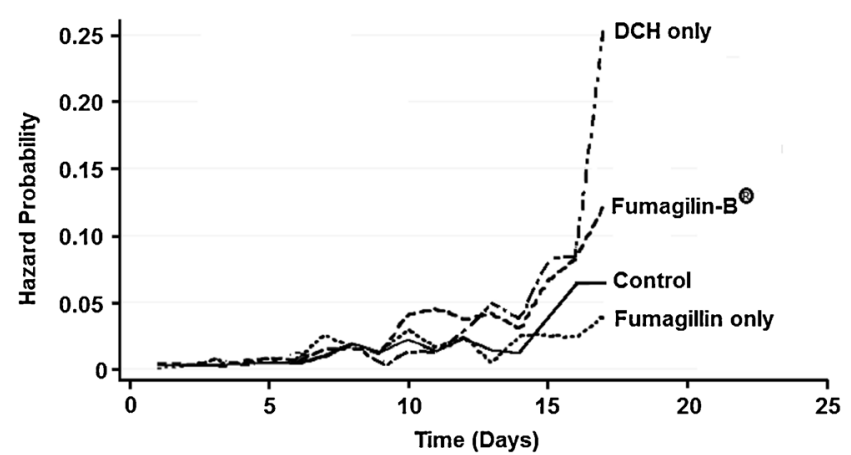

Figure 2. The predicted hazards from the complimentary log-log, Cox proportional hazards survival model for the cage trial to assess toxicity of dicyclohexylamine (DCH), fed ad libitum at a concentration of $40 \mu \mathrm{M}$ in $60 \%$ sugar solution, to bees infected with $N$. ceranae over 17 days of treatment. 
Table I. Results of the complimentary log-log, Cox proportional hazards survival model for the cage trial to assess toxicity of dicyclohexylamine (DCH), fed ad libitum at a concentration of $40 \mu \mathrm{M}$ in $60 \%$ sucrose solution, to bees infected with $N$. ceranae over 17 days of treatment

\begin{tabular}{llll}
\hline Treatment & HR & $P$ value & $95 \%$ CI \\
\hline \multicolumn{4}{l}{ DCH only vs. negative control } \\
Day 11 & 0.97 & 0.96 & $0.31-3.02$ \\
Day 13 & 3.66 & 0.01 & $1.48-9.02$ \\
Day 15 & 2.20 & 0.01 & $1.21-4.00$ \\
Day 16 & 1.36 & 0.24 & $0.81-2.28$ \\
Day 17 & 4.34 & $<0.01$ & $2.78-6.77$
\end{tabular}

Fumagilin-B $^{\circledR}$ vs. negative control

$\begin{array}{llll}\text { Day 11 } & 3.44 & 0.01 & 1.38-8.57 \\ \text { Day 13 } & 3.06 & 0.02 & 1.21-7.76 \\ \text { Day 15 } & 1.77 & 0.07 & 0.95-3.32 \\ \text { Day 16 } & 1.34 & 0.27 & 0.79-2.27 \\ \text { Day 17 } & 1.94 & 0.01 & 1.18-3.20\end{array}$

Fumagillin only vs. negative control

$\begin{array}{llll}\text { Day } 11 & 1.23 & 0.71 & 0.41-3.67 \\ \text { Day 13 } & 0.35 & 0.20 & 0.07-1.74 \\ \text { Day 15 } & 0.66 & 0.31 & 0.30-1.46 \\ \text { Day 16 } & 0.36 & 0.01 & 0.17-0.76 \\ \text { Day 17 } & 0.60 & 0.11 & 0.31-1.13\end{array}$

$\mathrm{DCH}$ only vs. fumagillin only

$\begin{array}{llll}\text { Day } 11 & 0.79 & 0.67 & 0.27-2.35 \\ \text { Day 13 } & 10.40 & <0.01 & 2.45-44.22 \\ \text { Day 15 } & 3.33 & <0.01 & 1.64-6.76 \\ \text { Day 16 } & 3.82 & <0.01 & 1.82-8.00 \\ \text { Day 17 } & 7.27 & <0.01 & 4.20-12.57\end{array}$

Fumagilin- $\mathrm{B}^{\circledR}$ vs. fumagillin only

$\begin{array}{llll}\text { Day 11 } & 2.79 & 0.02 & 1.18-6.60 \\ \text { Day 13 } & 8.70 & <0.01 & 2.01-37.66 \\ \text { Day 15 } & 2.68 & 0.01 & 1.29-5.58 \\ \text { Day 16 } & 3.77 & <0.01 & 1.79-7.93 \\ \text { Day 17 } & 3.25 & <0.01 & 1.80-5.89\end{array}$

Fumagilin-B ${ }^{\varpi}$ vs. DCH only

\begin{tabular}{llll} 
Day 11 & 3.53 & 0.01 & $1.42-8.79$ \\
Day 13 & 0.84 & 0.58 & $0.44-1.58$ \\
Day 15 & 0.80 & 0.41 & $0.48-1.35$ \\
Day 16 & 0.99 & 0.96 & $0.60-1.62$ \\
IDay 17 & 0.45 & $<0.01$ & $0.31-0.65$ \\
\hline
\end{tabular}

$H R$ hazard ratio, $C I$ confidence interval
Fumagilin-B ${ }^{\circledR}$ (fumagillin with DCH) was observed to have significantly higher bee mortality than any other group. By day 13, both Fumagilin$\mathrm{B}^{\circledR}$ and DCH alone had significantly higher mortality than either the control or purified fumagillin, but not from one another. Commercial Fumagilin$\mathrm{B}^{\circledR}$ and purified DCH consistently had higher bee mortality than purified fumagillin after day 11 . There were no significant differences in mortality between the purified fumagillin and the control group.

The results of the linear regression model for spore counts in the comparative cage trials to assess the effects of DCH are shown in Table II. The treatment was significantly associated with altered spore counts $(P \leq 0.01)$. The commercial Fumagilin- ${ }^{\circledR}$ reduced spore counts to zero in all replicates, which was a significant reduction (in the order of millions), compared to all other groups, including the purified fumagillin group $(P \leq 0.01$ for all). Purified fumagillin (no DCH) significantly reduced the spore count compared to the control and pure DCH groups $(P \leq 0.01$ for both); however, the spore reduction was in the order of 20 times less for both, which has questionable clinical significance. Residuals from the model were normally distributed and homoscedastic (data not shown).

\section{DISCUSSION}

It is important to remember that Fumagilin- $\mathrm{B}^{\circledR}$ is commercially sold as the dicyclohexylamine (DCH) salt, containing both fumagillin and $\mathrm{DCH}$ in a 1:1 stoichiometric ratio (Figure 1, compounds $\mathrm{a}$ and $\mathrm{b})$. Using Fumagilin- $\mathrm{B}^{\circledR}$ or Fumidil- ${ }^{\circledR}$ therefore introduces not only one, but two potentially biologically active compounds to the bee hive (van den Heever et al. 2014). We observed that the risk of bee mortality of $N$. ceranae -infected caged bees treated with DCH alone was not statistically different compared to the commercial Fumagilin-B ${ }^{\circledR}$ for most of the study period, with the exception of days 11 and 17 (Table II). Bees treated with DCH or commercial Fumagilin-B ${ }^{\circledR}$ had significantly higher risk of mortality compared with controls or those treated with purified fumagillin for most days after day 11, with a few exceptions (Table II). This indicates that the 
Table II. Results of linear regression model for the cage trial to assess the effects of various treatment preparations with or without dicyclohexylamine (DCH), fed ad libitum at a concentration of $40 \mu \mathrm{M}$ in $60 \%$ sugar solution, on spore counts in bees infected with $N$. ceranae over 17 days of treatment

\begin{tabular}{|c|c|c|c|c|c|}
\hline Compound & $\begin{array}{l}\text { Median spores } \\
\left(10^{6} \text { bee }\right)\end{array}$ & $\operatorname{SD}^{\mathrm{a}}\left(10^{6}\right)$ & $\begin{array}{l}\text { Coefficient } \\
\text { (spores/bee) }\end{array}$ & $P$ value & $95 \% \mathrm{CI}^{\mathrm{b}}$ \\
\hline Negative control & 12.0 & 3.72 & Referent & - & \\
\hline DCH only & 13.0 & 4.51 & 1 & 0.47 & -1 to 2 \\
\hline Fumagilin-B $^{\circledR}$ & 0 & 0 & $-12.38 \mathrm{e}^{+6}$ & $<0.01$ & $(-19.64$ to -7.91$) \mathrm{e}^{+6}$ \\
\hline Fumagillin only & 0.75 & 0.29 & -21 & $<0.01$ & -33 to -13 \\
\hline Fumagilin- $\mathrm{B}^{\circledR}$ vs. fumagillin only & - & - & $-0.59 \mathrm{e}^{+6}$ & $<0.01$ & $(-0.93$ to -0.37$) \mathrm{e}^{+6}$ \\
\hline Fumagilin-B ${ }^{\circledR}$ vs. DCH only & - & - & $-14.53 \mathrm{e}^{+6}$ & $<0.01$ & $(-23.05$ to -9.16$) \mathrm{e}^{+6}$ \\
\hline Fumagillin only vs. DCH only & - & - & -25 & $<0.01$ & -39 to -16 \\
\hline
\end{tabular}

$e$ exponent $\left(-12.38 \mathrm{e}^{+6}=-12,380,000\right)$

${ }^{\text {a }}$ Standard deviation

${ }^{\mathrm{b}}$ Confidence interval

observed bee mortality associated with the use of the commercial Fumagilin- ${ }^{\circledR}$ could likely be ascribed to the presence of $\mathrm{DCH}$ in the formulation. $\mathrm{DCH}$ has also been shown to be significantly more stable in honey under a variety of conditions (van den Heever et al. 2015a). When incurred honey samples were analyzed, these experimental observations were confirmed when it was found that $\mathrm{DCH}$ was present at significant concentrations in honey, even when no fumagillin is detectable (van den Heever et al. 2015a). In the present study, the beneficial properties of purified fumagillin were however observed to be almost 20 times lower than that of the commercial Fumagilin- ${ }^{\circledR}$. This is presumably due to the fact that the purified fumagillin is not chemically stabilized as a salt, and is therefore presumably prone to decomposition during handling and application.

Other hive products such as wax need to be examined for $\mathrm{DCH}$ contamination, since accumulation of DCH in the comb wax could negatively impact the development of young bee larvae, which are in close proximity to the wax comb at a stage of their lifecycle that make them more susceptible to the influence of substances like DCH. The environmental prevalence of DCH also needs to be established in order to ascertain if this route of contamination is indeed of concern not only for beekeeping, but also for human health (van den Heever et al. 2014).

Another aspect of DCH in apiculture that requires further study are the potential synergistic effects resulting from the combination of $\mathrm{DCH}$ and other known chemical contaminants in the hive. Previous research has shown that combined exposure to Fumagilin- $\mathrm{B}^{\circledR}$ and to tau -fluvalinate leads to an increase in tau-fluvalinate toxicity to honey bees, resulting in higher bee mortality (Johnson et al. 2013). Tau -fluvalinate was reportedly the most abundant pyrethroid found in North American apiaries in wax $(98.1 \%$ detection at a median concentration of $3595 \mathrm{ng} \mathrm{g}^{-1}$ ), pollen (88.3\% detection at a median concentration of $\left.5860 \mathrm{ng} \mathrm{g}^{-1}\right)$, and bees (83.6\% detection at a median concentration of $3595 \mathrm{ng} \mathrm{g}^{-1}$ ), according to a 2010 study (Mullin et al. 2010). The concentration of Fumagilin- ${ }^{\circledR}$ used for the synergism study (designed to evaluate sub therapeutic concentration effects) was however only $0.78 \mu \mathrm{M}$ (Johnson et al. 2013). which is 50 times less than the manufacturer's prescribed therapeutic dosage of $40 \mu \mathrm{M}$ for Fumagilin- ${ }^{\circledR}$, containing both fumagillin and $\mathrm{DCH}$ in equal amounts. This study indicated that a significant increase in toxic synergism with tau-fluvalinate can therefore be expected at the therapeutic concentration of Fumidil$\mathrm{B}^{\mathbb{B}}$ (or Fumagilin- $\mathrm{B}^{\mathbb{B}}$ ). It would be interesting to establish whether it is fumagillin or whether it is 
DCH that was responsible for the observed synergistic effect, since the commercially available Fumidil-B ${ }^{\circledR}$ was used in the synergism study (Johnson et al. 2013). Pyrethroids, including tau-fluvalinate, are reportedly also very stable in wax, where they can accumulate, with estimated half-lives of approximately 5 years (Bogdanov 2004). In previous reports, the half-life of DCH was observed as being approximately $1-3$ years in honey under various simulated conditions examined (van den Heever et al. 2015a). DCH can therefore be expected to accumulate in wax, and also to exhibit a long half-life in this matrix, but this remains to be confirmed. The continuous usage of Fumagilin- $\mathrm{B}^{\mathbb{B}}$ (and Fumidil- $\mathrm{B}^{\circledR}$ ) since almost 1950 (van den Heever et al. 2014). combined with its suspected prevalence and stability in wax, makes it highly likely that DCH will be present at elevated concentrations in this matrix. In light of this, and combined with our observations regarding the increased risk to bee mortality resulting from DCH exposure, it is important that the current analytical methodology be expanded to quantify DCH and fumagillin not only in honey, but also in other hive matrices such as in wax and perhaps in pollen. The synergistic effects of both $\mathrm{DCH}$ and fumagillin not only on tau-fluvalinate, but also on other frequently found chemical residues in the hive, warrants further research.

\section{CONCLUSIONS}

Evaluation of the toxicity of the $\mathrm{DCH}$, which is present in a 1:1 stoichiometric ratio to fumagillin in the commercial formulations of fumagillin, showed that DCH increases bee mortality. The genotoxic and mutagenic properties of $\mathrm{DCH}$, combined with its lipophilicity, could lead to its accumulation in comb wax, where it could potentially impact the development of the developing bee larvae and pupa. DCH is also a contaminant of concern in hive products, with regard to food safety and human health. The frequent detection of DCH residues in honey by LC-MS/MS, even in the absence of detectable fumagillin residues, has been previously reported (van den Heever et al. 2015b). The risk to the consumer associated with DCH residues in honey should be evaluated. DCH was also reported to be significantly more stable than fumagillin in honey (van den Heever et al. 2015a). A different formulation of fumagillin using a less toxic counter ion to form the salt would be beneficial. An example of such a counter ion could be meglumine, which is acceptable for human pharmaceutical use as a non-toxic counter ion that is used to crystallize carboxylic acid pharmaceuticals destined for human use. The use of a suitable non-toxic counter ion would eliminate $\mathrm{DCH}$ from the commercial fumagillin formulation and would clearly benefit bee health, as well as improve the quality of honey destined for human consumption.

L'effet de la dicyclohexylamine et de la fumagilline sur la mortalité d'abeilles (Apis mellifera) infectées par Nosema ceranae dans des essais en cage

Fumagilin-BC / fumagilline / DCH / toxicité / abeille domestique

Käfigversuche zum Effekt von Dicyclohexylamin and Fumagillin auf die Mortalität von Nosema ceranaeinfizierten Honigbienen (Apis mellifera)

Fumagilin-B ${ }^{\circledR} /$ Fumidil-B ${ }^{\circledR}$ / Fumagillin / Dicyclohexylamine / DCH / Toxizität / Honigbiene

\section{REFERENCES}

Bailey, L. (1953) Effect of fumagillin upon Nosema apis (Zander). Nature 171, 212-213

Bogdanov, S. (2004) Beeswax: quality issues today. Bee World 85, 46-50

Cantwell, G.E. (1970) Standard methods for counting nosema spores. Am. Bee J. 110, 222-223

Corrente, J.E., Chalita, L.V.A.S., Moreira, J.A. (2003) Choosing between Cox proportional hazards and logistic models for interval-censored data via bootstrap. J. Appl. Stat. 30, 37-57

Cox-Foster, D.L., Conlan, S., Holmes, E.C., Palacios, G., Evans, J.D., et al. (2007) A metagenomic survey of microbes in honey bee colony collapse disorder. Science 318, 283-287

Dohoo, I., Martin, W., Stryhn, H. (2009) Veterinary epidemiologic research. VER Inc., Charlottetown

Eble, T.E., Hanson, F.R. (1951) Fumagillin, an antibiotic from Aspergillus fumigatus $\mathrm{H}-3$. Antibiot. Chemother. 1, 54-58 
Fries, I., Feng, F., Silva, A.D., Slemenda, S.B., Pieniazek, N.J. (1996) Nosema ceranae n. sp. (Microspora, Nosematidae), morphological and molecular characterization of a microsporidian parasite of the Asian honey bee Apis cerana (Hymenoptera, Apidae). Eur. J. Prositol. 32, 356-365

Hanson, F.R., Eble, T.E. (1949) An antiphage agent isolated from Aspergillus sp. J. Bacteriol. 58, 527-529

Higes, M., Martín-Hernández, R., Botías, C., GarridoBailón, R., Gonzales-Porto, A.V., Barrios, L., Nozal, M.J., Bernal, J.L., Jimenez, J.J., Palencia, P.G., Meana, A. (2008) How natural infection by Nosema ceranae causes honeybee colony collapse. Environ. Microbiol. 10, 2659-2669

Higes, M., Martín-Hernández, R., Garrido-Bailón, E., Gonzalez-Porto, A.V., Garcia-Palencia, P., Meana, A., del Nozal, M.J., Mayo, R., Bernal, J.L. (2009) Honeybee colony collapse due to Nosema ceranae in professional apiaries. Environ. Microbiol. Rep. 1, 110-113

Higes, M., Nozal, M.J., Alvaro, A., Barrios, L., Meana, A., Martín-Hernández, R., Bernal, J.L., Bernal, J. (2011) The stability and effectiveness of fumagillin in controlling Nosema ceranae (Microsporidia) infection in honey bees (Apis mellifera) under laboratory and field conditions. Apidologie 42, 364-377

Johnson, R.M., Dahlgren, L., Siegfried, B.D., Ellis, M.D. (2013) Acaricide, fungicide and drug interactions in honey bees (Apis mellifera). PLoS ONE 8, e54092

Katznelson, H., Jamieson, C.A. (1952) Control of Nosema disease of honeybees with fumagillin. Science 115, 70-71

Martín-Hernández, R., Meana, A., Prieto, L., Salvador, A.M., Garrido-Bailón, E., Higes, M. (2007) Outcome of colonization of Apis mellifera by Nosema ceranae. Appl. Environ. Microbiol. 73, 6331-6338

Mullin, C.A., Frazier, M., Frazier, J., Ashcraft, S., Simonds, R., van Engelsdorp, D., Pettis, J.S. (2010) High levels of miticides and agrochemicals in North American apiaries: Implications for honey bee health. PLoS ONE 5, e9754

Stanimirović, Z., Aleksić, N., Kulić, M., Maletić, M. (2010) Fumagillin-induced chromosome aberrations in mouse bone-marrow cells. Arch. Biol. Sci. Belgrade. 62, 47-55
Stevanovic, J., Simeunovic, P., Gajic, B., Lakic, N., Radovic, D., Fries, I., Stanimirovic, Z. (2013) Characteristics of Nosema ceranae infection in Serbian honey bee colonies. Apidologie 44, 522-536

Thompson, G.J., Yockey, H., Lim, J., Oldroyd, B.P. (2007) Experimental manipulation of ovary activation and gene expression in honey bee (Apis mellifera) queens and workers: testing hypotheses of reproductive regulation. J. Exp. Zool. 307A, 600-610

van den Heever, J.P., Thompson, T.S., Curtis, J.M., Ibrahim, A., Pernal, S.F. (2014) Fumagillin: An overview of recent scientific advances and their significance for apiculture. J. Agric. Food Chem. 62, 27282737

van den Heever, J.P., Thompson, T.S., Curtis, J.M., Pernal, S.F. (2015a) Stability of dicyclohexylamine and fumagillin in honey. Food Chemistry 179, 152-158

van den Heever, J.P., Thompson, T.S., Curtis, J.M., Pernal, S.F. (2015b) Determination of dicyclohexylamine and fumagillin in honey by LC-MS/MS. Food Anal. Methods 8, 767-777

van den Heever, J.P., Thompson, T.S., Otto, S.J.G., Curtis, J.M., Ibrahim, A., Pernal, S.F. (2015c) Evaluation of Fumagilin-B ${ }^{\circledR}$ and other potential chemotherapies against Nosema ceranae-infected honey bees (Apis mellifera) in cage trial assays. Apidologie. doi:10.1007/s13592-015-0409-3

vanEngelsdorp, D., Evans, J.D., Saegerman, C., Mullin, C., Haubruge, E., Nguyen, B.K., Frazier, M., Frazier, J., Cox-Foster, D., Chen, Y., Underwood, R., Tarpy, D.R., Pettis, J.S. (2009) Colony collapse disorder: a descriptive study. PLoS ONE 4, e6481

Williams, G.R., Sampson, M.A., Shutler, D., Rogers, R.E.L. (2008) Does fumagillin control the recently detected invasive parasite Nosema ceranae in western honey bees (Apis mellifera )? J. Invertebr. Pathol. 99, 342-344

Williams, G.R., Shutler, D., Little, C.M., BurgherMacLellan, K.L., Rogers, R.E.L. (2011) The microsporidian Nosema ceranae, the antibiotic Fumagilin-B ${ }^{\circledR}$, and Western honey bee (Apis mellifera ) colony strength. Apidologie 42, 15-22

Zander, E. (1909) Tierische Parasiten als Krankenheitserreger bei der Biene. Münchener Bienenzeitung 31, 196-204 